\title{
TITLE:
}

\section{Dynamics of traveling waves under spatiotemporal forcing}

AUTHOR(S):

Ohta, T; Tokuda, $\mathrm{H}$

CITATION:

Ohta, T ... [et al]. Dynamics of traveling waves under spatiotemporal forcing. Physical Review E 2005, 72(4): 046216.

ISSUE DATE:

2005-10

URL:

http://hdl.handle.net/2433/49962

RIGHT:

Copyright 2005 American Physical Society 
PHYSICAL REVIEW E 72, 046216 (2005)

\title{
Dynamics of traveling waves under spatiotemporal forcing
}

\author{
Takao Ohta and Hidekazu Tokuda \\ Yukawa Institute for Theoretical Physics, Kyoto University, Kyoto 606-8502, Japan
}

(Received 28 April 2004; published 24 October 2005)

\begin{abstract}
We study dynamics of traveling waves under spatiotemporal forcing in nonequilibrium systems. Based on the model equations where phase separation and chemical reactions take place simultaneously so that traveling waves are formed in a self-organized manner, we apply a space-time dependent external force. Entrainment and modulation of traveling waves are investigated numerically in one dimension. We develop a theoretical analysis to understand the dynamics obtained.
\end{abstract}

DOI: 10.1103/PhysRevE.72.046216

PACS number(s): 89.75.Kd, 82.40.Ck, 05.45.-a

\section{INTRODUCTION}

Various self-organized patterns emerge in nonequilibrium open systems. Spatiotemporal structures of these patterns have been studied extensively both experimentally and theoretically for many years [1-3]. The effects of spatially uniform external forcing have also been investigated with tremendous interest due to their potential application for nano/ mesoscopic domain control in material sciences. For instance, photoinduced waves in liquid-crystalline monolayers have been studied [4-6]. A global delayed feedback method has been developed to investigate spatiotemporal pattern formation by controlling catalytic $\mathrm{CO}$ oxidation in an oscillatory surface chemical reaction [7].

This problem also has an important implication in biological sciences such as coherent dynamics of interacting cells and nonlinear stimuli-response phenomena of chemical networks in a living cell. In this respect, there are a number of studies of synchronization of nonlinear coupled oscillators with or without a uniformly oscillating external field $[1,8]$.

However, there are less extensive studies for the effects of spatiotemporal forcing in nonuniform systems having a spatially regular structure. Experiments of convective nematic fluid under spatially periodic forcing have been performed [9] and a theoretical study has been available [10]. Influence of temporal modulation on pattern formation has been investigated in convective fluids [11-13] and in chemical reactions $[14,15]$. Only recently, experiments and theoretical consideration have began for Turing patterns influenced by spatiotemporal forcing. For example, effects of illuminating light on a spatially periodic structure are investigated in chemically reacting systems [16-20].

The purpose of the present paper is to investigate, theoretically and by numerical simulations, dynamics of traveling waves under spatiotemporal external forcing. In our previous papers, we introduced a model set of equations for phase separated mixtures undergoing chemical reactions [21,22] and studied the synchronization and modulation of motionless and propagating waves in two dimensions by applying spatially uniform oscillating external disturbance [23]. The external forcing was imposed by allowing one of the reaction rates to be time dependent. Here we generalize this study to the case of spatiotemporal external forcing and carry out numerical simulations of dynamics of traveling waves in one dimension and develop a theoretical analysis to understand the results of simulations. To our knowledge, despite the previous studies mentioned above, response of propagating periodic structures to the space-time dependent external disturbance has not been explored so far. In the present paper, we consider the case that the spatial period of the external forcing is the same as the intrinsic period of the traveling waves. The incommensurate case will be published separately.

The organization of the present paper is as follows. In the next section, we start with a brief explanation of the model system and the linear stability analysis of the uniform equilibrium solution. In Sec. III, we present the dynamical phase diagram obtained numerically. To see when the entrainment is broken, we develop a theory based on the amplitude equation for traveling waves. The special case where the external frequency is equal to zero, i.e., motionless external forcing, is investigated both by numerical simulations and theoretically in Sec. IV. The discussion is given in Sec. V. In the Appendix, an alternative method of studying the stability limit of entrainment is provided in terms of phase dynamics.

\section{MODEL EQUATION AND LINEAR STABILITY}

In our previous studies, we introduced the model system of phase separation undergoing chemical reactions [21-23]. This mixture is composed of three chemical components $A$, $B$, and $C$ where $A$ and $B$ species tend to segregate each other and there is a cyclic chemical reaction

$$
A \stackrel{\gamma_{1}}{\rightarrow} \stackrel{\gamma_{2}}{\longrightarrow} \underset{\stackrel{\gamma_{3}}{\longrightarrow}}{\rightarrow} A
$$

with the reaction rates $\gamma_{1}, \gamma_{2}$, and $\gamma_{3}$. We assume that other components are also involved in the chemical reaction, which are supplied to the system and removed from the system sufficiently rapidly so that they are constant in both space and time. Therefore these components modify only the reaction rates.

By introducing the local concentrations $\psi_{A}, \psi_{B}$, and $\psi_{C}$ of $A, B$, and $C$ components respectively, the time-evolution equations are given by [21]

$$
\frac{\partial \psi}{\partial t}=\nabla^{2} \frac{\delta F}{\delta \psi}+f(\psi, \phi)
$$




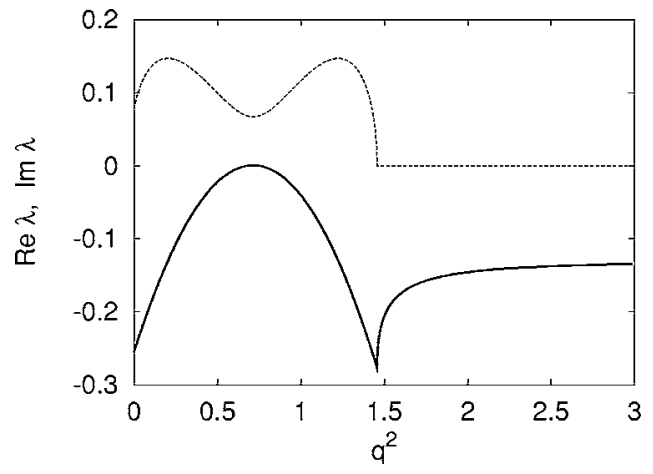

FIG. 1. The wave-number dependence of $\operatorname{Re} \lambda(q)$ (solid line) and $\operatorname{Im} \lambda(q)$ (dashed line) for $D=1.0, \tau=1.46, \gamma_{1}=0.3, \gamma_{2}=0.16$, and $\gamma_{3}=0.05$. The vertical and horizontal axes are dimensionless.

$$
\frac{\partial \phi}{\partial t}=g(\psi, \phi)
$$

where $\psi=\psi_{A}-\psi_{B}$ and $\phi=\psi_{A}+\psi_{B}$. We have imposed the condition $\psi_{A}+\psi_{B}+\psi_{C}=1$ which is justified by the assumption of the uniformity of other chemical species as mentioned above. The free energy functional $F$ is given by

$$
F=\int d \mathbf{r}\left[\frac{D}{2}(\nabla \psi)^{2}-\frac{\tau}{2} \psi^{2}+\frac{1}{4} \psi^{4}\right],
$$

where $D$ and $\tau$ are positive constants. Generally speaking, there are coupling terms between $\psi$ and $\phi$ in the free energy, e.g., through the $\phi$ dependence of $\tau$. However, for the sake of simplicity, we have ignored such a nonlinearity. The last terms in Eqs. (2) and (3) arise from the chemical reaction (1) and are given, respectively, by

$$
\begin{gathered}
f(\psi, \phi)=-\left(\gamma_{1}+\frac{\gamma_{2}}{2}\right) \psi-\left(\gamma_{1}-\frac{\gamma_{2}}{2}+\gamma_{3}\right) \phi+\gamma_{3}, \\
g(\psi, \phi)=\frac{\gamma_{2}}{2} \psi-\left(\frac{\gamma_{2}}{2}+\gamma_{3}\right) \phi+\gamma_{3} .
\end{gathered}
$$

It is noted that the diffusion term is not considered in Eq. (3). We have verified that the diffusion of $\phi$ does not alter essentially the dynamics described below [22].

By putting $f=g=0$, the uniform stationary solution of Eqs. (2) and (3) are readily obtained as

$$
\begin{aligned}
& \psi_{0}=\frac{\gamma_{3}\left(\gamma_{2}-\gamma_{1}\right)}{\gamma_{1} \gamma_{2}+\gamma_{2} \gamma_{3}+\gamma_{3} \gamma_{1}}, \\
& \phi_{0}=\frac{\gamma_{3}\left(\gamma_{2}+\gamma_{1}\right)}{\gamma_{1} \gamma_{2}+\gamma_{2} \gamma_{3}+\gamma_{3} \gamma_{1}} .
\end{aligned}
$$

To examine the linear stability of the uniform solution we put $\psi-\psi_{0}=c_{1} \exp (\lambda t+i q x)$ and $\phi-\phi_{0}=c_{2} \exp (\lambda t+i q x)$ with $c_{1}$ and $c_{2}$ constants and substitute into Eqs. (2) and (3). In this section, we fix the parameters as $D=1, \gamma_{1}=0.3$, and $\gamma_{3}$ $=0.05$ and the remaining two parameters $\tau$ and $\gamma_{2}$ are varied. At some range of the parameters, the eigenvalue is complex. An example is shown in Fig. 1 for $\tau=1.46$ and $\gamma_{2}=0.16$ as a function of wave number. Note that the real part becomes

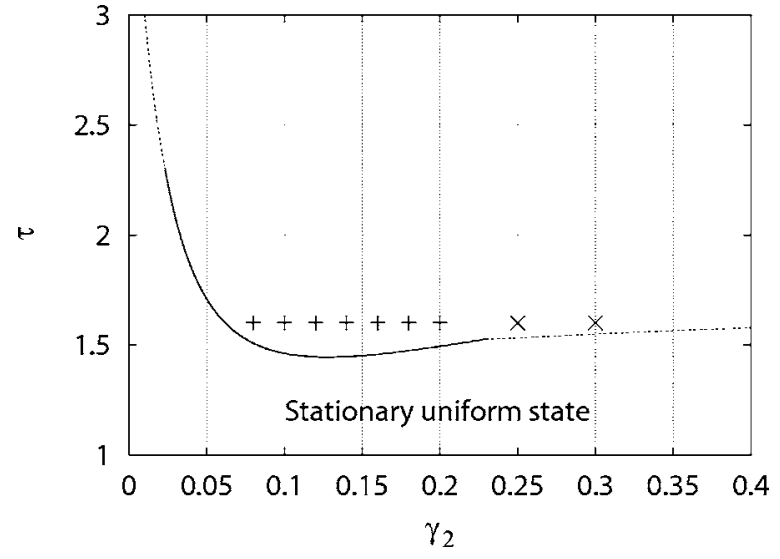

FIG. 2. Bifurcation diagram for the uniform stationary solution for $D=1, \gamma_{1}=0.3$, and $\gamma_{3}=0.05$. The full line and the dotted line are the Hopf bifurcation line and the Turing-type bifurcation line, respectively. A traveling wave appears at the parameters indicated by the symbol + whereas a motionless pattern at the symbol $\times$.

positive at a finite wave number $q_{c}$ and the imaginary part has a minimum at $q=q_{c}$. The latter is a general property of the present model system. The expressions of $q_{c}, \tau_{c}$, and the critical frequency $\omega_{c}$ which is the imaginary part of the eigenvalue at $q=q_{c}$ are given for $\tau>3 \psi_{0}^{2}$ by

$$
\begin{gathered}
q_{c}=\left(\frac{\tau-3 \psi_{0}^{2}}{2}\right)^{1 / 2}, \\
\tau_{c}=3 \psi_{0}^{2}+2\left(\gamma_{1}+\gamma_{2}+\gamma_{3}\right)^{1 / 2}, \\
\omega_{c}=\left(\frac{\gamma_{1} \gamma_{2}-\gamma_{2} \gamma_{3}-\gamma_{2}^{2}}{2}-\gamma_{3}^{2}\right)^{1 / 2} .
\end{gathered}
$$

The linear stability analysis gives us the bifurcation diagram as shown in Fig. 2 [22]. The value of $\tau$ at the Hopf bifurcation point for $\gamma_{2}=0.16$ is $\tau_{c} \approx 1.46$ at which the critical wave number is $q_{c} \approx 0.9$ and the critical frequency $\omega_{c}$ $\approx 0.07$. Just at the Hopf bifurcation line, a pair of the eigenfunctions of the linearized operator gives us

$$
\begin{gathered}
\psi(x, t)=\Psi \cos \left( \pm q_{c} x-\omega_{c} t\right), \\
\phi(x, t)=\Phi \cos \left( \pm q_{c} x-\omega_{c} t+\theta\right),
\end{gathered}
$$

where the amplitudes $\Psi$ and $\Phi$ are not determined within the linear theory and the phase difference is given by

$$
\theta=\tan ^{-1}\left(\frac{2 \omega_{c}}{\gamma_{2}+2 \gamma_{3}}\right)
$$

which yields $\theta \approx 0.494$ for $\gamma_{2}=0.16$.

In order to study the behavior above the bifurcation lines we have carried out numerical simulations of Eqs. (2) and (3) in one dimension. The Euler method is employed with the time increment 0.001 and by dividing the space into 128 cells with the mesh size 0.5 . The linear dimension of the system is $L=64$ which is chosen to be as commensurate as 


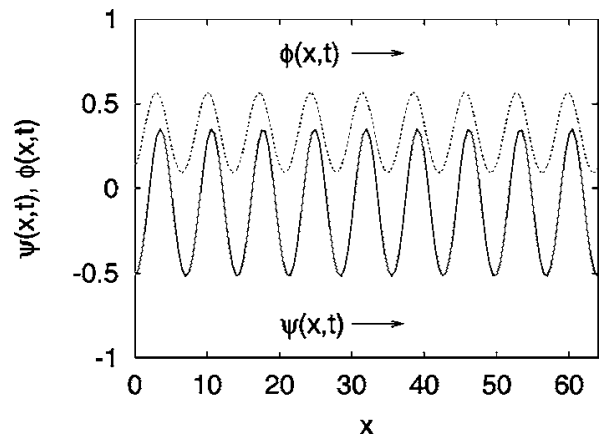

FIG. 3. Spatial profiles of $\psi(x, t)$ (solid line) and $\phi(x, t)$ (dashed line) for $D=1.0, \tau=1.6, \gamma_{1}=0.3, \gamma_{2}=0.16$, and $\gamma_{3}=0.05$. Both $\psi(x, t)$ and $\phi(x, t)$ are propagating to the right at the same velocity.

possible with the spatial period $2 \pi / q_{c} \approx 7$ at the bifurcation point. In this set of the parameters, there are nine wave trains under the periodic boundary condition.

A motionless periodic pattern appears in the region indicated by $\times$ in Fig. 2 whereas a propagating wave pattern appears in the region + . It is emphasized that the motionless solution is not due to the Turing mechanism but its origin is related to microphase separation in block copolymers [24]. This is the reason why the diffusion term can be omitted in Eq. (3) to obtain a motionless periodic structure. In order to emphasize this property, we shall call the bifurcation a Turing-type bifurcation to distinguish it from the ordinary Turing instability.

Figure 3 shows the spatial variation of a propagating wave. Since Eqs. (2) and (3) are invariant under the transformation $x \rightarrow-x$, the traveling wave can propagate either to the right or to the left with the proper phase difference between $\phi$ and $\psi$. Hereafter we choose (with an appropriate initial condition) a wave traveling to the right without loss of generality. The phase difference is in good agreement with the theoretical result given by Eq. (14).

It is mentioned that a similar self-organized formation of traveling waves has also been studied both theoreticaly and experimentally in adsorbed molecules on metals $[25,26]$.

\section{PHASE DIAGRAM}

The spatiotemporal forcing is added to Eqs. (2) and (3) as

$$
\Gamma(x, t)=\epsilon \cos \left(q_{f} x-\Omega t\right) .
$$

This is a sinusoidal force traveling to the right at the velocity $\Omega / q_{f}$. Here we suppose that the system is exposed through a periodically arrayed slit by illuminating light and the slit moves at a constant velocity $\Omega / q_{f}$. As a result, we assume that the reaction rate $\gamma_{3}$ is modified such that $\gamma_{3} \rightarrow \gamma_{3}+\Gamma$. We have ignored a term $\Gamma \phi$ arising from the $\gamma_{3} \phi$ term in Eqs. (5) and (6) providing a sufficiently small forcing $\epsilon$.

Under these conditions, the model equations (2) and (3) can be written as

$$
\begin{aligned}
\frac{\partial \psi}{\partial t}= & \nabla^{2}\left[-\nabla^{2} \psi-\tau \psi+\psi^{3}\right]+a_{1} \psi+a_{2} \phi \\
& +\epsilon \cos \left(q_{f} x-\Omega t\right)+a_{3},
\end{aligned}
$$

$$
\frac{\partial \phi}{\partial t}=b_{1} \psi+b_{2} \phi+\epsilon \cos \left(q_{f} x-\Omega t\right)+b_{3}
$$

where

$$
\begin{gathered}
a_{1}=-\left(\gamma_{1}+\frac{\gamma_{2}}{2}\right), \\
a_{2}=-\left(\gamma_{1}-\frac{\gamma_{2}}{2}+\gamma_{3}\right), \\
a_{3}=\gamma_{3}, \\
b_{1}=\frac{\gamma_{2}}{2}, \\
b_{2}=-\left(\frac{\gamma_{2}}{2}+\gamma_{3}\right), \\
b_{3}=\gamma_{3} .
\end{gathered}
$$

In the present paper, we confine ourselve to the case that the spatial period of the external forcing is the same as that of traveling waves, i.e., $q_{f}=q_{c}$. The case $q_{f} \neq q_{c}$ will be published separately. Hereafter, the parameters are fixed as $\tau$ $=1.6$ and $\gamma_{2}=0.16$. The actual spatial period for $\tau=1.6$ in the absence of the external forcing is difficult to evaluate analytically. However, since the value of $\tau$ is close to the critical value $\tau_{c}$, we assume that the actual wave number of the traveling wave is not much different from $q_{c}$.

The dynamical behaviors of the traveling waves obtained by changing the external frequency and the force strength $\epsilon$ are summarized in Fig. 4. This phase diagram is obtained by the asymptotic behavior of the traveling wave after imposing the external force. The region indicated by + shows that the traveling wave is entrained with the external force so that it propagates at the velocity $\Omega / q_{c}$. When the frequency $\Omega$ of the external forcing is much different from $\omega_{c}$ as in the region indicated by the diamond symbols, entrainment breaks down and the traveling wave is modulated such that the amplitude as well as the propagating velocity is uniformly oscillating. When the velocity is decreased, the amplitude is also decreased and vice versa. We have verified numerically that the frequency of the modulation is proportional to $\mid \Omega$ $-\omega_{c} \mid$.

In the region for sufficiently small external frequency indicated by the white inverse triangle the wave undergoes an oscillatory motion. That is, the wave trains propagate to the right and to the left alternatively and gradually move to the right as a whole. In the region of the white triangle at $\Omega$ $=0.01$ and $\epsilon=0.005$, the wave propagates to the left oscillating its amplitude despite the fact that the external forcing moves to the right. This phenomenon is observed in the following process. First, we provide a traveling wave propagating to the right and then turn on the external force. The traveling wave is reflected by the periodic external force and changes the propagating direction. Although this reflection of traveling waves is found only for one set of the parameters, the result is reproducible. These respective behaviors are 


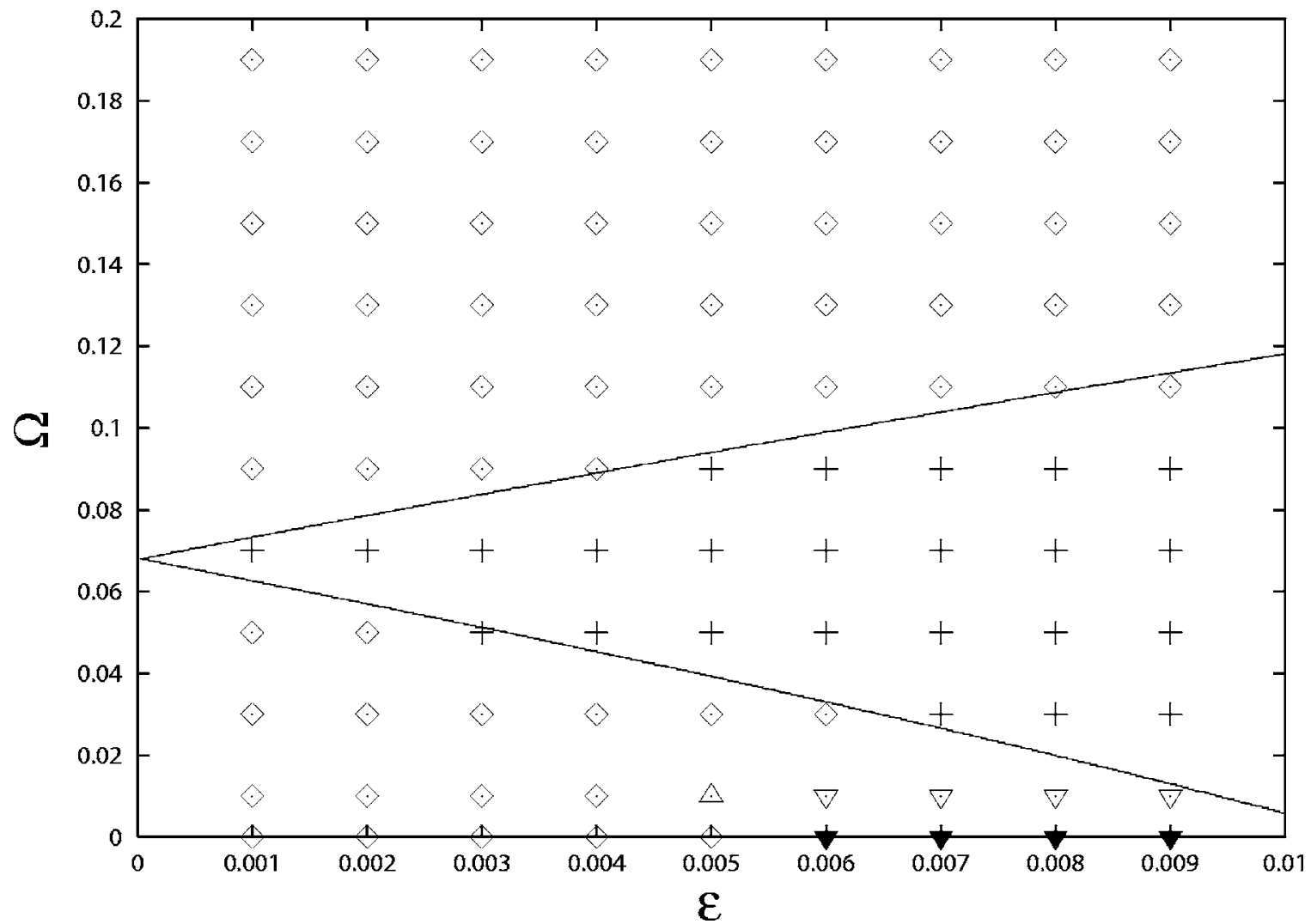

FIG. 4. Phase diagram for $\gamma_{2}=0.16, \tau=1.6$, and $q_{f}=q_{c}$. The solid line is the theoretically obtained stability limit of the unmodulated traveling wave having the same velocity as the external force. The meanings of the symbols are given in the text.

shown in the space-time plot of the concentration $\psi$ in Fig. 5.

In the region indicated by a black triangle for static external force, i.e., $\Omega=0$, waves are trapped by the force $(\psi$ is large in the region where the force is small) and move back and forth repeatedly in the confined interval of one wavelength of the external force. The results for $\Omega=0$ will be described in further detail in the next section.

Now we perform a theoretical analysis to understand the dynamics. When the external forcing is not considered, the amplitude equation near the Hopf bifurcation has been derived from Eqs. (2) and (3) [22]. When the external force is weak as in the present study, the amplitude equation is modified as

$$
\frac{\partial W_{R}}{\partial t}=A W_{R}+B \frac{\partial^{2} W_{R}}{\partial x^{2}}-g\left|W_{R}\right|^{2} W_{R}+\epsilon^{\prime} e^{i\left(\Omega-\omega_{c}\right) t},
$$

where we are concerned, without loss of generality, only with a wave traveling to the right, whose amplitude is de-

(a)

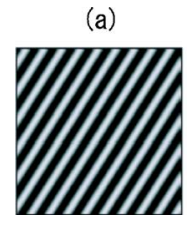

(b) (c)
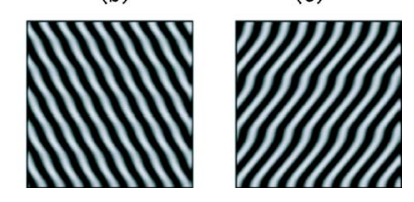

FIG. 5. (Color online) Space (horizontal)-time (vertical) plot of $\psi$ for (a) $\epsilon=0.006$ and $\Omega=0.07$, (b) $\epsilon=0.005$ and $\Omega=0.01$, and (c) $\epsilon=0.006$ and $\Omega=0.11$. The value of $\psi$ is large (small) for lighter (darker) regions. noted by $W_{R}$. This complex amplitude is defined through

$$
\left(\begin{array}{c}
\psi \\
\phi
\end{array}\right)=\vec{U} W_{R} e^{-i q_{c} x+i \omega_{c} t}+\vec{U}^{*} W_{R}^{*} e^{i q_{c} x-i \omega_{c} t}
$$

where $X^{*}$ is the complex conjugate of $X$ and $\vec{U}$ is the eigenvector corresponding to the eigenvalue $i \omega_{c}$ at the Hopf bifurcation point and is given by

$$
\vec{U}=\left(\begin{array}{c}
1 \\
\frac{b_{1}}{i \omega_{c}-b_{2}}
\end{array}\right) .
$$

All the coefficients in Eq. (19) are complex, which we write as $A=A_{1}+i A_{2}$. The coefficient $\epsilon^{\prime}$ is proportional to the strength $\epsilon$ in Eq. (15). These are given by

$$
A_{1}=\frac{\left(\tau-\tau_{c}\right) q_{c}^{2}}{2}
$$

$$
\begin{gathered}
A_{2}=\frac{\left(\tau-\tau_{c}\right) b_{2} q_{c}^{2}}{2 \omega_{c}}, \\
B_{1}=2 q_{c}^{2}, \\
B_{2}=\frac{2 b_{2} q_{c}^{2}}{\omega_{c}},
\end{gathered}
$$




$$
\begin{gathered}
g_{1}=\frac{3}{2} q_{c}^{2}\left(1+24 r_{1} \psi_{0}^{2} q_{c}^{2}-\frac{24 b_{2} r_{2} \psi_{0}{ }^{2} q_{c}{ }^{2}}{\omega_{c}}\right), \\
g_{2}=\frac{3}{2} q_{c}^{2}\left(24 r_{2} \psi_{0}^{2} q_{c}^{2}+\frac{b_{2}+24 b_{2} r_{1} \psi_{0}^{2} q_{c}^{2}}{\omega_{c}}\right), \\
\epsilon_{1}^{\prime}=\frac{\epsilon}{2}\left(\frac{a_{2}^{2}+a_{2} b_{2}}{a_{2}^{2}+b_{2}^{2}+\omega_{c}^{2}}\right), \\
\epsilon_{2}^{\prime}=\frac{\epsilon}{2}\left(\frac{a_{2} \omega_{c}}{a_{2}^{2}+b_{2}^{2}+\omega_{c}^{2}}\right) .
\end{gathered}
$$

For the typical set of the parameters chosen in the present paper, the real part of these coefficients is positive whereas the imaginary part is negative. The constants $r_{1}$ and $r_{2}$ in Eqs. (26) and (27) are defined, respectively, by

$$
\begin{aligned}
& r_{1}=\frac{3\left(3 a_{1} b_{2}^{2}+3 b_{2}^{3}+12 a_{1} \omega_{c}^{2}+11 b_{2} \omega_{c}^{2}\right)}{\left(9 a_{1} b_{2}+9 b_{2}^{2}-3 \omega_{c}^{2}\right)^{2}+\left[18 \omega_{c}\left(a_{1}+b_{2}\right)\right]^{2}}, \\
& r_{2}=\frac{6 \omega_{c}^{3}}{\left(9 a_{1} b_{2}+9 b_{2}^{2}-3 \omega_{c}^{2}\right)^{2}+\left[18 \omega_{c}\left(a_{1}+b_{2}\right)\right]^{2}} .
\end{aligned}
$$

A remark is now in order. The amplitude equation for a traveling wave generally has a term $v \partial W_{R} / \partial x$ with $v$ a group velocity [10]. However, such a term does not exist in Eq. (19) because $\partial \omega / \partial q=0$ at $q=q_{c}$ as shown in Fig. 1.

We examine the stability of the uniform stationary solution of Eq. (19). Putting $W_{R}$ as $W_{R}=R e^{i \theta}$ where $R=Q+r(t)$ and $\theta=\theta_{0}+\left(\Omega-\omega_{c}\right) t+\delta(t)$ with $r(t)$ and $\delta(t)$ small deviations, the stationary solution satisfies

$$
\begin{gathered}
A_{1} Q-g_{1} Q^{3}+\epsilon_{1}^{\prime} \cos \left(\theta_{0}\right)+\epsilon_{2}^{\prime} \sin \left(\theta_{0}\right)=0, \\
\Omega-\omega_{c}=A_{2}-g_{2} Q^{2}+\frac{\epsilon_{2}^{\prime}}{Q} \cos \left(\theta_{0}\right)-\frac{\epsilon_{1}^{\prime}}{Q} \sin \left(\theta_{0}\right) .
\end{gathered}
$$

Eliminating $\theta_{0}$ from these equations, we obtain

$$
Q^{2}\left(A_{1}-g_{1} Q^{2}\right)^{2}+Q^{2}\left(\Omega-\omega_{c}^{R}\right)^{2}=\epsilon_{1}^{\prime 2}+\epsilon_{2}^{\prime 2},
$$

where we have defined

$$
\omega_{c}^{R}=\omega_{c}+A_{2}-g_{2} Q^{2} .
$$

Linearization of Eq. (19) with respect to $r(t)$ and $\delta(t)$ gives us

$$
\begin{gathered}
\frac{\partial r}{\partial t}=\left(A_{1}-3 g_{1} Q^{2}\right) r+\left[\epsilon_{2}^{\prime} \cos \left(\theta_{0}\right)-\epsilon_{1}^{\prime} \sin \left(\theta_{0}\right)\right] \delta, \\
\frac{\partial \delta}{\partial t}=\frac{1}{Q^{2}}\left[\epsilon_{1}^{\prime} \sin \left(\theta_{0}\right)-\epsilon_{2}^{\prime} \cos \left(\theta_{0}\right)\right] r-2 g_{2} Q r \\
-\frac{1}{Q}\left[\epsilon_{1}^{\prime} \cos \left(\theta_{0}\right)+\epsilon_{2}^{\prime} \sin \left(\theta_{0}\right)\right] \delta .
\end{gathered}
$$

The eigenvalues of this set of equations are given by

$$
\lambda=A_{1}-2 g_{1} Q^{2} \pm \lambda_{2},
$$

where (a)

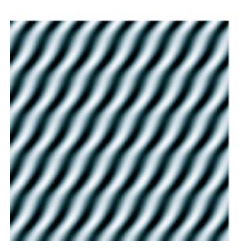

(b)

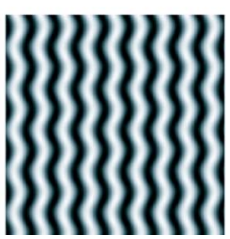

(c)

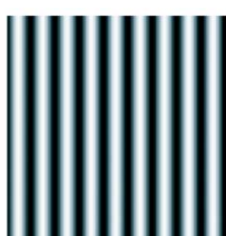

FIG. 6. (Color online) Space (horizontal)-time (vertical) plot of $\psi$ for (a) $\epsilon=0.005$, (b) $\epsilon=0.01$, and (c) $\epsilon=0.02$. The parameters are chosen as $\Omega=0, \tau=1.6$, and $\gamma_{2}=0.16$.

$\lambda_{2}=\left[Q^{4}\left(g_{1}^{2}-3 g_{2}^{2}\right)+\left(A_{2}+\omega_{c}-\Omega\right)\left(4 Q^{2} g_{2}-A_{2}-\omega_{c}+\Omega\right)\right]^{1 / 2}$.

When $\epsilon_{1}^{\prime 2}+\epsilon_{2}^{\prime 2} \ll 1$ and $\left|\Omega-\omega_{c}^{R}\right| \gg 1$, this is approximated as

$$
\lambda \simeq A_{1}-2 g_{1} Q^{2} \pm i\left|\Omega-\omega_{c}^{R}\right| .
$$

We have solved numerically Eq. (34) for a given set of the parameters and searched the condition such that the real part of $\lambda$ given by Eq. (38) becomes positive. The solid lines in Fig. 4 are obtained in this way and are the stability limit of a completely entrained traveling wave. It is found that agreement between the simulations and the theory is almost perfect except for the larger values of $\epsilon$. This might be attributed to the approximation of weak external forcing in the amplitude Eq. (19). It is emphasized that there are no adjustable parameters in the above comparison. Note that the eigenvalue is as complex as Eq. (40) indicating that this is a Hopf bifurcation. This is consistent with the observed oscillatory modulation of traveling waves beyond the threshold.

An alternative way to evaluate the entrainment limit is to derive the phase equation of motion starting with Eq. (19). This is given in the Appendix. We have verified numerically that the result is equivalent with the condition $\lambda>0$ in Eq. (40).

Before closing this section, we mention that the average velocity of the modulated waves for larger values of $\Omega$ is almost precisely equal to the intrinsic velocity given by $\omega_{c} / q_{c}$. However, the wave velocity for smaller values of $\Omega$ is smaller than the intrinsic velocity. For example, the wave velocity at $\Omega=0.01$ and $\epsilon=0.004$ is 0.068 whereas $\omega_{c} / q_{c}$ $=0.078$.

\section{TRAPPED OSCILLATION FOR $\Omega=0$}

In this section, we show the results for $\Omega=0$. That is, the external forcing is motionless and spatially periodic. When the strength of the external forcing $\epsilon$ is small, waves can propagate modulating the amplitude and the velocity periodically as shown in the phase diagram 4 . The space-time plot of $\psi$ in this case is displayed in Fig. 6(a). However, beyond a certain critical value $\epsilon=\epsilon_{1}{ }^{*} \approx 0.0055$ for $\tau=1.6$ and $\gamma_{2}$ $=0.16$, the waves cannot propagate any more but undergo a coherent oscillation trapped by the external force as shown in Fig. 6(b). There is another threshold of $\epsilon=\epsilon_{2}{ }^{*} \approx 0.0141$ above which the waves do not move but they are frozen as is evident in Fig. 6(c). These transitions of the wave property will be investigated theoretically below. 
(a)

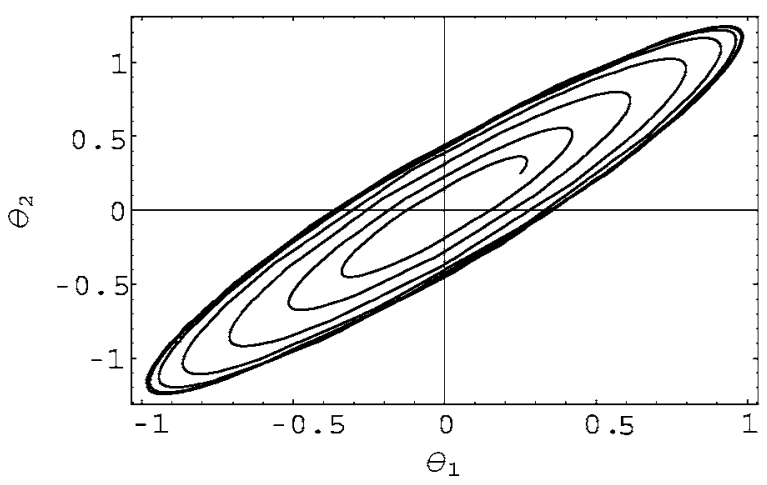

(b)

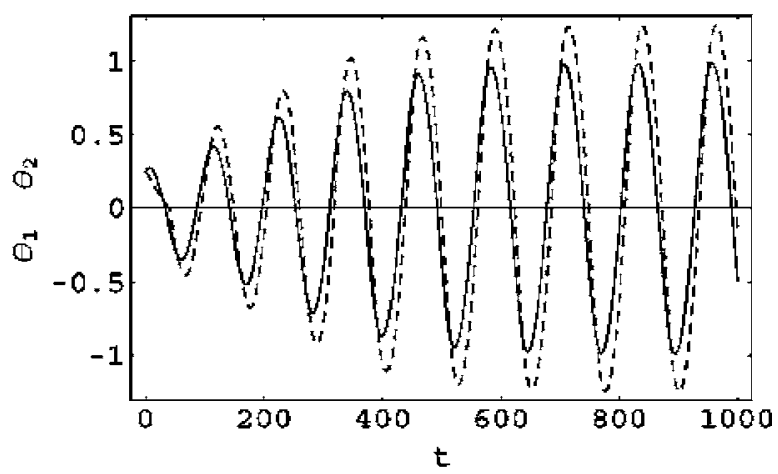

FIG. 7. (a) Limit cycle oscillation of $\theta_{1}$ and $\theta_{2}$ for $\epsilon=0.01$ and (b) time evolution of $\theta_{1}$ (solid line) and $\theta_{2}$ (dashed line) for $\epsilon=0.01$ obtained numerically from Eqs. (47) and (48).

We shall show that the trapped oscillation can be understood by a phase dynamics approach. As mentioned above, numerical simulations reveal that Eqs. (16) and (17) with $\Omega=0$ have a motionless periodic solution for sufficiently large values of $\epsilon$. What we examine here is the stability of this stationary solution by decreasing $\epsilon$. We put

$$
\begin{aligned}
& \psi=\psi_{0}+\psi_{1} \cos \left(q_{c} x\right), \\
& \phi=\phi_{0}+\phi_{1} \cos \left(q_{c} x\right) .
\end{aligned}
$$

We have verified that the harmonic variation of $\psi$ and $\phi$ is a good approximant of the simulation results. Numerical simulations indicate that the spatial variation of $\psi$ and $\phi$ is anitphase with respect to the external spatial modulation $\epsilon \cos \left(q_{c} x\right)$. This implies that one has to choose the negative solution of $\psi_{1}$ and $\phi_{1}$.

Substituting Eqs. (41) and (42) into Eqs. (16) and (17) and ignoring higher harmonics, we obtain

$$
-q_{c}^{4} \psi_{1}+\tau q_{c}^{2} \psi_{1}-q_{c}^{2}\left(3 \psi_{0}^{2} \psi_{1}+\frac{3}{4} \psi_{1}^{3}\right)+a_{1} \psi_{1}+a_{2} \phi_{1}+\epsilon=0
$$

$$
b_{1} \psi_{1}+b_{2} \phi_{1}+\epsilon=0 \text {. }
$$

This gives us the motionless periodic solutions $\psi_{1}=\bar{\psi}_{1}$ and $\phi_{1}=\bar{\phi}_{1}$. As mentioned above, these are negative. In order to study the stability we introduce the time-dependent phase variables as

$$
\begin{aligned}
& \psi=\psi_{0}+\bar{\psi}_{1} \cos q_{c}\left[x+\theta_{1}(t)\right], \\
& \phi=\phi_{0}+\bar{\phi}_{1} \cos q_{c}\left[x+\theta_{2}(t)\right] .
\end{aligned}
$$

Substituting Eqs. (45) and (46) into Eqs. (16) and (17) and multiplying the resultant equations, respectively, by $\sin q_{c}[x$ $\left.+\theta_{1}(t)\right]$ and $\sin q_{c}\left[x+\theta_{2}(t)\right]$, and performing the integral over $x$, we obtain

$$
q_{c} \frac{d \theta_{1}}{d t}=c_{11} \sin q_{c}\left(\theta_{1}-\theta_{2}\right)+c_{12} \sin \left(q_{c} \theta_{1}\right)
$$

$$
q_{c} \frac{d \theta_{2}}{d t}=-c_{21} \sin q_{c}\left(\theta_{2}-\theta_{1}\right)+c_{22} \sin \left(q_{c} \theta_{2}\right),
$$

where

$$
\begin{gathered}
c_{11}=-\frac{a_{2} \bar{\phi}_{1}}{\bar{\psi}_{1}}, \quad c_{12}=-\frac{\epsilon}{\bar{\psi}_{1}}, \\
c_{21}=\frac{b_{1} \bar{\psi}_{1}}{\bar{\phi}_{1}}, \quad c_{22}=-\frac{\epsilon}{\bar{\phi}_{1}} .
\end{gathered}
$$

It is noted that these coefficients are positive. Linearizing the set of equations (47) and (48) around the stationary solution $\theta_{1}=\theta_{2}=0$, we obtain the eigenvalue equation

$$
\lambda^{2}-\left(c_{11}+c_{12}-c_{21}+c_{22}\right) \lambda+c_{11} c_{22}-c_{21} c_{12}+c_{12} c_{22}=0 \text {. }
$$

The Hopf instability condition is

$$
c_{11}+c_{12}-c_{21}+c_{22}>0
$$

and

$$
c_{11} c_{22}-c_{21} c_{12}+c_{12} c_{22}>0 .
$$

We have verified that the condition (53) is always satisfied. The condition (52) yields $\epsilon_{2}{ }^{*}=0.01404$ which agrees quite well with the numerical simulations $\epsilon_{2}{ }^{*}=0.0141$. For $\epsilon$ $<\epsilon_{2}{ }^{*}$, the set of equations (47) and (48) exhibits a limit cycle oscillation as shown in Fig. 7. The oscillations themselves of $\psi$ and $\phi$ are found to be quantitatively in agreement with the simulations (though not shown) and hence the lowest mode expansion in Eqs. (41) and (42) is justified self-consistently.

If the value of $\epsilon$ is further decreased, the limit cycle oscillation disappears and it turns out that $\theta_{1}$ and $\theta_{2}$ increase (or decrease) monotonously as shown in Fig. 8. This behavior corresponds to the modulated traveling wave shown in Fig. 6(a). This occurs for $\epsilon$ smaller than $\epsilon \approx 0.0044$, which is slightly smaller than the other bifurcation threshold $\epsilon_{1}^{*}$ $=0.0055$. The origin is attributed to the fact that the approximation used in the derivation of Eqs. (47) and (48) becomes worse for smaller values of $\epsilon$. In fact, the time-dependence of $\psi_{1}$ and $\phi_{1}$ is not considered. Nevertheless, the theory repro- 


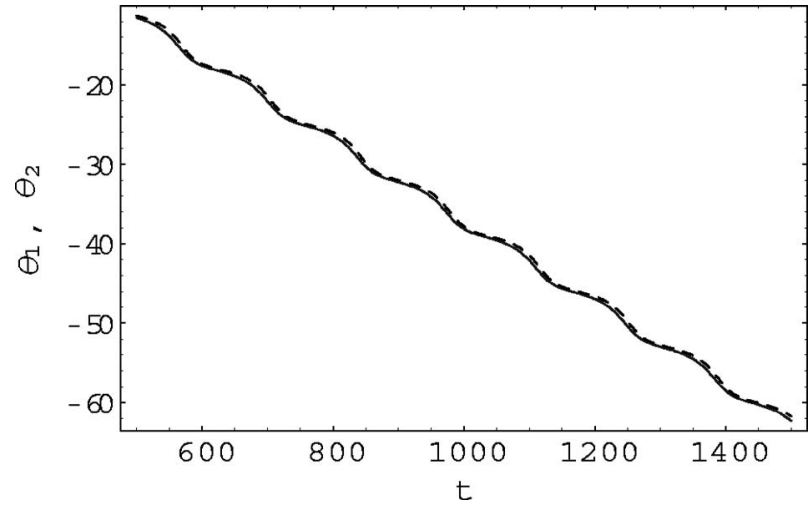

FIG. 8. Time evolution of $\theta_{1}$ (solid line) and $\theta_{2}$ (dashed line) for $\epsilon=0.004$ obtained numerically from Eqs. (47) and (48).

duces the simulations qualitatively and the global dynamics for $\Omega=0$ are understood almost completely.

\section{DISCUSSION}

In the preceding sections, we have investigated, for the first time, entrainment and modulation of a traveling wave under external forcing which depends on both space and time. The dynamical phase diagram has been obtained numerically. Although we restrict ourselves to the case such that the spatial period of the external force is the same as that of traveling waves, we have found several nontrivial dynamics. (1) When $\Omega \approx \omega_{c}$, the wave trains entrain with the external forcing. (2) When $\Omega$ is much different from $\omega_{c}$, this entrainment breaks down. (3) When external forcing is sufficiently strong, the traveling waves are pinned. (4) When the forcing is intermediate, trapped oscillations of wave trains are observed. We have performed a theoretical analysis based on the amplitude equation and the phase equations.

We emphasize that our traveling waves are spontaneously formed without external forcing whereas those in Ref. [18] are induced by the external forcing. As a result, there is an essential difference in the amplitude equation. In our case, the coefficients of the amplitude equation (19) are complex whereas those are real in Ref. [18] as well as in more recent studies $[19,20]$. There is one similarity in both studies. That is, the spatially uniform modulation of waves as in Fig. 5(c) appears also in Ref. [18]. However, more interesting dynamics such as the trapped oscillations can never exit in the system in Ref. [18]. Emergence of the traveling wave in the opposite direction to the external forcing due to reflection is also a characteristic feature of the present system.

Although the present study starts with the specific model system, we believe that the results are quite general for modulation dynamics of spontaneously formed traveling waves independent of the details of the model equations. This will be confirmed if one develops a theory such as phase dynamics to describe the modulated waves beyond the linear stability analysis. In fact, the coupled phase dynamics given by Eqs. (47) and (48) provides us with a general theory of trapped oscillations of domains under external force. It is our hope that the present study will stimulate experiments for control of self-organized dynamic patterns far from equilibrium by using external forcing.

\section{ACKNOWLEDGMENTS}

This work was supported by the Grant-in-Aid for the 21st Century COE "Center for Diversity and Universality in Physics" from the Ministry of Education, Culture, Sports, Science and Technology (MEXT) of Japan.

\section{APPENDIX}

In this Appendix, we derive the condition of entrainment of the traveling waves with the external forcing. Because we consider the case $q_{f}=q_{c}$ and the spatially uniform modulations, we omit the diffusion term in Eq. (19)

$$
\frac{\partial W_{R}}{\partial t}=A W_{R}-g\left|W_{R}\right|^{2} W_{R}+\xi e^{i\left(\Omega-\omega_{c}\right) t+i \delta},
$$

where we have put

$$
\epsilon^{\prime}=\xi e^{i \delta},
$$

with $\xi=\left(\epsilon_{1}^{\prime 2}+\epsilon_{2}^{\prime 2}\right)^{1 / 2}$ and $\tan \delta=\epsilon_{2}^{\prime} / \epsilon_{1}^{\prime}$. Putting $W_{R}=R e^{i \theta}$, we obtain

$$
\begin{gathered}
\frac{\partial R}{\partial t}=A_{1} R-g_{1} R^{3}+\xi \cos \left[\left(\Omega-\omega_{c}\right) t+\delta-\theta\right], \\
\frac{\partial \theta}{\partial t}=A_{2}-g_{2} R^{2}+\frac{\xi}{R} \sin \left[\left(\Omega-\omega_{c}\right) t+\delta-\theta\right] .
\end{gathered}
$$

The amplitude $R$ is split into the equilibrium solution and its deviation as

$$
R=R_{0}+r(t)
$$

where $R_{0}=\left(A_{1} / g_{1}\right)^{1 / 2}$ and the deviation $r$ obeys

$$
\frac{\partial r}{\partial t}=-2 A_{1} r-3 g_{1} R_{0} r^{2}-g_{1} r^{3}+\xi \cos \left[\left(\Omega-\omega_{c}\right) t+\delta-\theta\right] .
$$

Because the amplitude relaxes much faster than the phase, we may approximate $\partial r / \partial t=0$ so that we have up to the lowest order of $\xi$

$$
r=\frac{\xi}{2 A_{1}} \cos \left[\left(\Omega-\omega_{c}\right) t+\delta-\theta\right] .
$$

Substituting this into Eq. (57), we have a closed equation for the phase variable

$$
\frac{\partial \eta}{\partial t}=-\left(\Omega-\omega_{c}^{R}\right)-\alpha \sin (\eta+\Theta)
$$

where $\omega_{c}^{R}$ has been defined by Eq. (35) and we have introduced

$$
\eta(t)=\theta(t)-\left(\Omega-\omega_{c}\right) t-\delta .
$$

The constants $\alpha$ and $\Theta$ are defined by 


$$
\begin{gathered}
\alpha=\frac{\xi}{R_{0}}\left(1+\frac{g_{2}^{2}}{g_{1}^{2}}\right)^{1 / 2}, \\
\Theta=\tan ^{-1}\left(\frac{g_{2}}{g_{1}}\right) .
\end{gathered}
$$

Equation (61) implies that when $\left|\Omega-\omega_{c}^{R}\right|<\alpha$, there is a stable equilibrium solution $\eta=0$ which corresponds to the complete entrainment with the external forcing. When $\mid \Omega$ $-\omega_{c}^{R} \mid>\alpha$, this entrainment breaks down. This condition should be equivalent with $\lambda>0$ in Eq. (38). However, we have not tried to prove this equivalence because the expression is complicated, but we verified numerically that these two conditions agree with each other quite precisely. From Eqs. (A7) and (A9), we obtain

$$
r(t)=\frac{\xi}{2 A_{1}} \cos \eta(t) .
$$

Therefore, although $|\eta|$ increases in time for $\left|\Omega-\omega_{c}^{R}\right|>\alpha$, the amplitude oscillates within a finite interval.
[1] Y. Kuramoto, Chemical Oscillation, Waves and Turbulance (Springer-Verlag, Berlin, 1984).

[2] D. Walgraef, Spatio-Temporal Pattern Formation (SpringerVerlag, New York, 1997).

[3] M. C. Cross and P. C. Hohenberg, Rev. Mod. Phys. 65, 851 (1993).

[4] Y. Tabe and H. Yokayama, Langmuir 11, 4609 (1995).

[5] R. Reigada, F. Sagues, and A. S. Mikhailov, Phys. Rev. Lett. 89, 038301 (2002).

[6] T. Okuzono, Y. Tabe, and H. Yokoyama, Phys. Rev. E 69, 050701(R) (2004).

[7] M. Bertram, C. Beta, M. Pollmann, A. S. Mikhailov, H. H. Rotermund, and G. Ertl, Phys. Rev. E 67, 036208 (2003).

[8] Synchronization: Theory and application, edited by A. Pikovsky and Y. Maistrenko (Kluwer, London, 2003).

[9] M. Lowe and J. P. Gollub, Phys. Rev. A 31, 3893 (1985).

[10] P. Coullet, Phys. Rev. Lett. 56, 724 (1986).

[11] C. W. Meyer, D. S. Cannell, G. Ahlers, J. B. Swift, and P. C. Hohenberg, Phys. Rev. Lett. 61, 947 (1988).

[12] P. C. Hohenberg and J. B. Swift, Phys. Rev. A 35, 3855 (1987).

[13] H. Riecke, J. D. Crawford, and E. Knobloch, Phys. Rev. Lett. 61, 1942 (1988).
[14] V. Petrov, Q. Oyang, and H. L. Swinney, Nature (London) 388, 655 (1997).

[15] K. Martinez, A. L. Lin, R. Kharrazian, X. Sailer, and H. L. Swinney, Physica D 168-169, 1 (2002).

[16] A. P. Muñuzuri, M. Dolink, A. M. Zhabotinsky, and I. R. Epstein, J. Am. Chem. Soc. 121, 8065 (1999).

[17] V. K. Vanag, A. M. Zhabotinsky, and I. R. Epstein, Phys. Rev. Lett. 86, 552 (2001).

[18] S. Rüdiger, D. G. Míguez, A. P. Muñuzuri, F. Sagués, and J. Casademunt, Phys. Rev. Lett. 90, 128301 (2003).

[19] D. G. Míguez, E. M. Nicola, A. P. Muñuzuri, J. Casademunt, F. Sagués, and L. Kramer, Phys. Rev. Lett. 93, 048303 (2004).

[20] F. Sagués, D. G. Míguez, E. M. Nicola, A. P. Muñuzuri, J. Casademunt, and L. Kramer, Physica D 199, 235 (2004).

[21] T. Okuzono and T. Ohta, Phys. Rev. E 64, 045201(R) (2001).

[22] T. Okuzono and T. Ohta, Phys. Rev. E 67, 056211 (2003).

[23] S. Sugiura, T. Okuzono, and T. Ohta, Phys. Rev. E 66, 066216 (2002).

[24] M. Motoyama and T. Ohta, J. Phys. Soc. Jpn. 66, 2715 (1997).

[25] A. von Oertzen, H. H. Rotermund, A. S. Mikhailov, and G. Etrl, J. Phys. Chem. B 104, 3155 (2000).

[26] M. Hildebrand, A. S. Mikhailov, and G. Ertl, Phys. Rev. Lett. 81, 2602 (1998). 